\title{
Encyclopedia of Geropsychology
}



Nancy A. Pachana

Editor

\section{Encyclopedia of Geropsychology}

With 148 Figures and 100 Tables

算 Springer 
Editor

Nancy A. Pachana

The University of Queensland

Brisbane, QLD, Australia

ISBN 978-981-287-081-0

ISBN 978-981-287-082-7 (eBook)

ISBN 978-981-287-083-4 (print and electronic bundle)

DOI 10.1007/978-981-287-082-7

Library of Congress Control Number: 2016953014

(C) Springer Science+Business Media Singapore 2017

This work is subject to copyright. All rights are reserved by the Publisher, whether the whole or part of the material is concerned, specifically the rights of translation, reprinting, reuse of illustrations, recitation, broadcasting, reproduction on microfilms or in any other physical way, and transmission or information storage and retrieval, electronic adaptation, computer software, or by similar or dissimilar methodology now known or hereafter developed.

The use of general descriptive names, registered names, trademarks, service marks, etc. in this publication does not imply, even in the absence of a specific statement, that such names are exempt from the relevant protective laws and regulations and therefore free for general use.

The publisher, the authors and the editors are safe to assume that the advice and information in this book are believed to be true and accurate at the date of publication. Neither the publisher nor the authors or the editors give a warranty, express or implied, with respect to the material contained herein or for any errors or omissions that may have been made.

Printed on acid-free paper

This Springer imprint is published by Springer Nature

The registered company is Springer Nature Singapore Pte Ltd.

The registered company address is: 152 Beach Road, \#22-06/08 Gateway East, Singapore 189721, Singapore 


\section{Foreword: The Frontiers of Geropsychology}

In Undaunted Courage, the late historian, Stephen Ambrose (1996) chronicled the challenges faced by Meriwether Lewis as he set out on the Lewis and Clark expedition. He and his band of explorers were emissaries of President Thomas Jefferson, seeking a northwest passage and exploring the western territories of a young nation. Throughout the expedition, Lewis sent scouts back to President Jefferson, reporting on the landscape and the flora and fauna of the young nation's largely unknown territory.

In many ways, the entries of this comprehensive encyclopedia are like Lewis's scouts reports, only the territory being described is at once universal and immediately personal: the psychology of aging. Although concerns about aging and the latter part of the life span can be traced to the ancient Greeks (Abeles 2015), G. Stanley Hall's (1922) Senescence: The Last Half of Life marked psychology's formal acknowledgment of the relevance of later life for psychology and psychology's relevance for understanding that portion of the lifespan. In the almost one hundred years since Hall's publication, the field of geropsychology has expanded tremendously in breadth and depth.

When someone asks "What does psychology have to do with aging?" there are simple and complex answers. The simple answer: "Lots!"

Paul Baltes (1987, 1997; Baltes et al. 2007) outlined a more complex answer. He suggested that psychological models of adult development and aging had to account for four key elements: multidirectionality; plasticity; the historical context; and multiple causation. Baltes reminded us that aging includes both growth and decline (a lesson highlighted in Freund et al. (2016) entry). He also highlighted that compensatory skills can be learned to accommodate changing abilities. (Kuhn and Lindenberger (2016) would later differentiate plasticity from flexibility, a differentiation found in Wahl and Wettstein's (2016) contribution to this encyclopedia.) Baltes's emphasis on the historical context was a reminder of the influence of cohort and historical moments on individuals and societies, a theme echoed in Kennison, et al.'s (2016) entry. Finally, by highlighting multiple causation, Baltes focused our attention on the interaction between and among influences that shape the development and expression of psychological functioning, including biological and psychological elements (again, reflected in Riffin and Loeckenhoff's (2016) entry).

Editor Nancy Pachana is to be commended for the range of talented scholars and important topics she has assembled in this encyclopedia; both 
are impressive. Together, hundreds of scholars have shared their expertise to report on the state of the art in geropsychology in the early twenty-first century. Along the way, they have demonstrated range of methods (observational and experimental), design (longitudinal, cross-sectional, cohort-sequential), and measurement strategies (intensive, repeated measures; single time surveys; etc.) Highlighting both inter- and intra-individual differences in rates and processes of aging, they have expanded Baltes's outline and helped us answer three important questions: How do psychological processes affect aging? How does aging affect psychological processes? How do the contexts of individuals affect the interaction of aging and psychological processes?

Throughout the encyclopedia, various psychological processes are highlighted for their impact on the processes of aging: for example, resilience (Staudinger and Greve 2016); the positivity effect (Reed and Carstensen 2016); social cognition (von Hippel et al. 2016); and social exchange (Wan and Antonucci 2016).

Conversely, some have focused on the impact of aging processes on psychological and social functions: for example, cognition (Schaie and Willis 2016); executive function (Karbach and Unger 2016); attention (Ruthruff and Lien 2016); memory (Zimprich and Kurtz 2016); decision-making (Mata 2016); personality (Helmes 2016; Diehl and Brother 2016); sexuality (Connaughton and McCabe 2016); and sexual orientation (Kimmel 2016).

At the same time, the contributors have focused on the impact of various contexts on the interplay of aging and psychological functioning: for example, social policy (Lum and Wong 2016); advocacy (DiGilio and Elmore 2016); technology (Lane et al. 2016); and work and retirement (Desmette and Fraccaroli 2016).

This encyclopedia will be a resource for many audiences: students of gerospsychology who seek an introduction to the methods and findings of the field; teachers and scholars who seek insightful summaries of the complex literatures encompassed by geropsychology; and clinicians who are involved in translational research and service, extending the implications of basic research paradigms into the lives of aging adults, their families, and their communities.

The encyclopedia's "scholar scouts" of the territory of aging, who include the very capable associate editors of this text, have given us detailed reports on both the process and substance of exploring the territory. They allow us to understand aging in new ways and to see new prospects and new challenges in a territory we thought we knew. They also remind us of how far we have come in understanding the very human experience of aging. Savor the journey.

\section{References}

Abeles, N. (2015). Historical perspectives on clinical geropsychology. In P. Lichtenberg \& B. T. Mast (Eds.). APA handbook of clinical geropsychology: Vol. 1. History and status of the field and perspectives on aging (pp. 3-17). Washington: American Psychological Association. doi: 10.1037/14458-02. 
Ambrose, S. E. (1996). Undaunted courage: Meriwether Lewis, Thomas Jefferson, and the opening of the American West. New York: Simon \& Schuster.

Baltes, P. B. (1987). Theoretical propositions of life-span developmental psychology: On the dynamics between growth and decline. Developmental Psychology, 23, 611-626.

Baltes, P. B. (1997). On the incomplete architecture of human ontogeny: Selection, optimization as foundation of developmental theory. American Psychologist, 52, 366-380.

Baltes, P. B., Lindenberger, U., \& Staudinger, U. M. 2007. Life span theory in developmental psychology. In Handbook of child psychology (Vol. I, p. 11). doi: 10.1002/9780470147658.chpsy0111.

Connaughton, C., \& McCabe, M. (2016). Sexuality and aging. In N. Pachana (Ed.), Encyclopedia of geropsychology. New York: Springer.

Desmette, D., \& Fraccaroli, F. (2016). From work to retirement. In N. Pachana (Ed.), Encyclopedia of geropsychology. New York: Springer.

Diehl, M., \& Brother, A. (2016). Self theories of the aging person. In N. Pachana (Ed.), Encyclopedia of geropsychology. New York: Springer.

Freund, A., Napolitano, C., \& Knecht, M. (2016). Life management through selection, optimization, and compensation. In N. Pachana (Ed.), Encyclopedia of geropsychology. New York: Springer.

Hall. G. (1922). Senescence: The last half of life. New York: Appleton. doi: $10.1037 / 10896-000$.

Helmes, E. (2016). Stage theories of personality. In N. Pachana (Ed.), Encyclopedia of geropsychology. New York: Springer.

Karbach, J., \& Unger, K. (2016). Executive functions. In N. Pachana (Ed.), Encyclopedia of geropsychology. New York: Springer.

Kennison, R., Situ, D., Reyes, N., \& Ahacic, K. (2016). Cohort effects. In N. Pachana (Ed.), Encyclopedia of geropsychology. New York: Springer.

Kimmel, D. (2016). History of sexual orientation and geropsychology. In N. Pachana (Ed.), Encyclopedia of geropsychology. New York: Springer.

Kühn, S., \& Lindenberger, U. (2016). Research on human plasticity in adulthood: A lifespan agenda. In K. W. Schaie \& S. L. Willis (Eds.), Handbook of the psychology of aging (8th ed., pp. 105-123). Amsterdam: Academic Press. doi: 10.1016/B978-0-12-411469-2.00006-6.

Lum, T., \& Wong, G. (2016). Social policies for aging societies. In N. Pachana (Ed.), Encyclopedia of geropsychology. New York: Springer.

Matas, R. (2016). Decision making. In N. Pachana (Ed.), Encyclopedia of geropsychology. New York: Springer.

Riffin, C., \& Loeckenhoff, C. (2016). Life span developmental psychology. In N. Pachana (Ed.), Encyclopedia of geropsychology. New York: Springer.

Ruthruff, E., \& Lien, M. (2016). Aging and attention. In N. Pachana (Ed.), Encyclopedia of geropsychology. New York: Springer.

Schaie, K. W., \& Willis, S. (2016). History of cognitive aging research. In N. Pachana (Ed.), Encyclopedia of geropsychology. New York: Springer.

Staudinger, U., \& Greve, W. (2016). Resilience and aging. In N. Pachana (Ed.), Encyclopedia of geropsychology. New York: Springer. 
Wahl, H., \& Wettstein, M. (2016). Plasticity of aging. In N. Pachana (Ed.), Encyclopedia of geropsychology. New York: Springer.

Zimprich, D., \& Kurtz, T. (2016). Process and systems views of aging and memory. In N. Pachana (Ed.), Encyclopedia of geropsychology. New York: Springer.

Michael A. Smyer

Professor of Psychology

Bucknell University 


\section{Preface}

Geropsychology is a relatively young field which spans a range of topic areas covering a subject of perennial interest to researchers, practitioners, and lay persons - namely, the psychology of later life. This book aims to thoroughly cover the main subtopics within the field of geropsychology, including historical and theoretical perspectives, clinical and applied geropsychology, cognitive and experimental geropsychology, geriatric neuropsychology and neuroscience, social geropsychology, health perspectives in geropsychology, work and retirement in later life, and longitudinal aging and centenarian studies.

The aim is to cover all aspects of geropsychology in a comprehensive way, with an international perspective and attention paid to both established and emerging topics in the field. The illustrations and high quality of the images, as well as the breadth of topics covered, will be key to its success.

In recent years, several advances in theory, measurement, and application across these many areas within geropsychology, coupled with innovations in domains ranging from genetics to social media and the Internet, have dramatically expanded the field. Simultaneously, the aging of the population in the developing and the developed world has enlivened interest in geropsychology. I hope that this book serves as a timely addition to the growing body of literature on this topic.

Brisbane - Australia

Nancy A. Pachana

December 2016 



\section{Acknowledgments}

The Encyclopedia of Geropsychology has been a truly international collaborative effort. I would like to thank all of the wonderful researchers across the globe who contributed entries to this work, my subsection editors for their diligence and creativity, the patience and support of all of the staff at Springer, the encouragement of my colleagues, friends, and family, and the love and support of my husband Tim. 



\section{About the Editor}

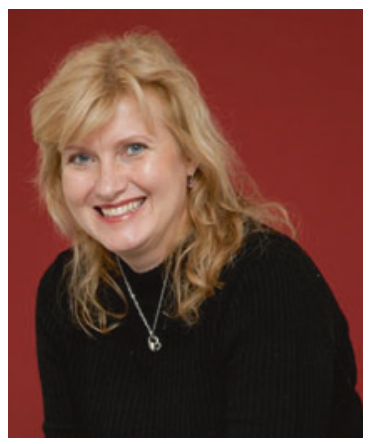

Nancy A. Pachana

School of Psychology

The University of Queensland

Brisbane, Queensland, Australia

Dr. Nancy A. Pachana is a clinical geropsychologist, neuropsychologist, and professor in the School of Psychology at The University of Queensland and is codirector of the UQ Ageing Mind Initiative, providing a focal point for clinical, translational aging-related research at UQ. She has an international reputation in the area of geriatric mental health, particularly with her research on late-life anxiety disorders. She is codeveloper of the Geriatric Anxiety Inventory, a published brief self-report inventory in wide clinical and research use globally, translated into over two dozen languages. She has published over 200 peer-reviewed articles, book chapters, and books on various topics in the field of aging and has been awarded more than $\$ 20$ million in competitive research funding, primarily in the areas of dementia and mental health in later life. Her research is well cited and she maintains a clear international focus in her collaborations and research interests, which include anxiety in later life, psychological interventions for those with Parkinson's disease, nursing home interventions, driving safety and dementia, teaching and learning in psychogeriatrics, and mental health policy and aging.

Nancy was elected a Fellow of the Academy of Social Sciences in Australia in 2014. She is also a Fellow of the Australian Psychological Society and is the recipient of numerous prizes and awards, including an Australian Davos Connection Future Summit Leadership Award, for leadership on aging issues in Australia. She serves on the editorial boards of several journals, including the Journals of Gerontology: Psychological Science, one of the top two journals in the world for publication of research in the science of the psychology of aging. Originally from the United States, Nancy was awarded her A.B. from Princeton University in 1987, her Ph.D. from Case Western Reserve University in 1992, and completed postdoctoral fellowships at the Neuropsychiatric Institute at UCLA, Los Angeles, and the Palo Alto Veterans Medical Center, Palo Alto, California. She is an avid bird watcher and photographer and an intrepid traveller. 



\section{Section Editors}

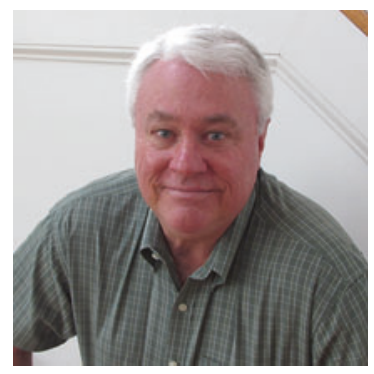

Christopher Hertzog School of Psychology, Georgia Institute of Technology, Atlanta, USA

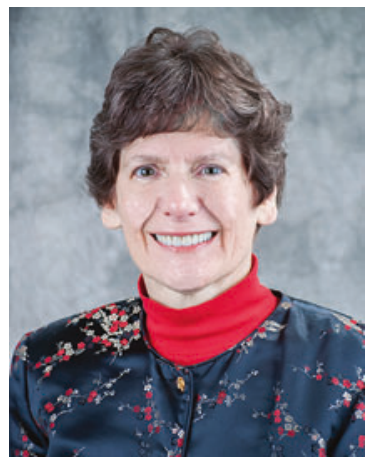

Marcia G. Ory Health Promotion and Community Health Sciences, School of Public Health, Texas A\&M Health Science Center Texas, Texas, USA 


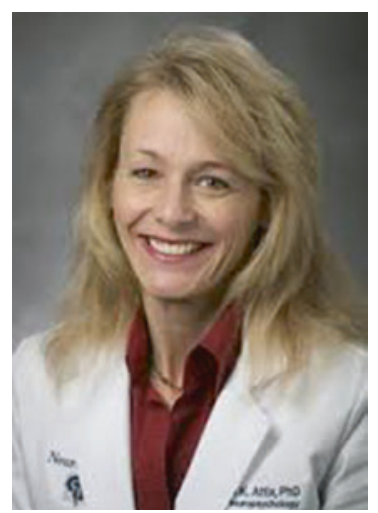

Deborah Attix Duke Clinical Neuropsychology Service, Department of Psychiatry and Behavioral Sciences, Department of Neurology, Duke University Medical Center, Durham, USA

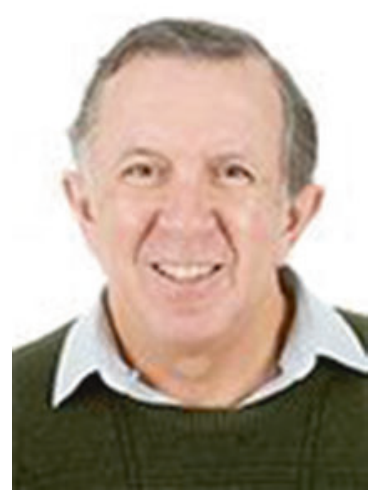

Bob G. Knight School of Psychology and Counseling, University of Southern Queensland, Toowoomba, Queensland, Australia

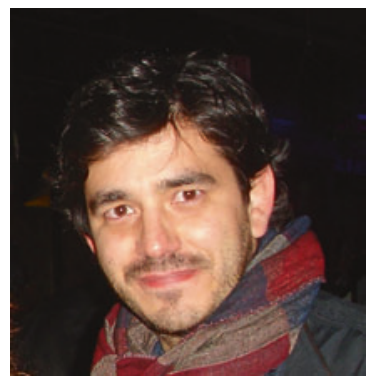

Óscar Ribeiro Institute of Biomedical Sciences Abel Salazar of the University of Porto (UNIFAI \& CINTESIS), University of Aveiro, Department of Education and Psychology, Higher Institute of Social Service of Porto, Porto, Portugal 


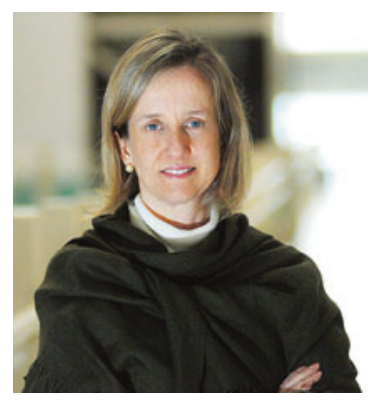

Mônica Yassuda Gerontology and Neurology Departments, University of São Paulo, São Paulo, Brazil

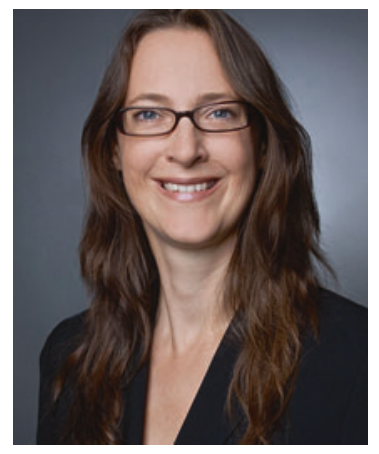

Daniela S. Jopp University of Lausanne, Institute of Psychology, Géopolis, Lausanne, Switzerland

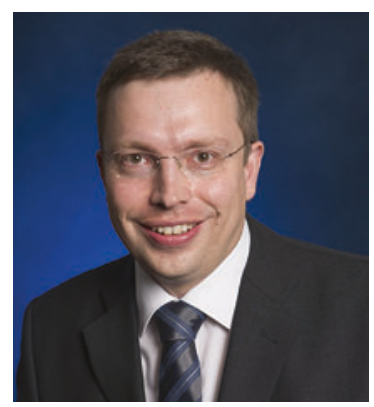

Hannes Zacher School of Management, Queensland University of Technology, Brisbane, Queensland, Australia 


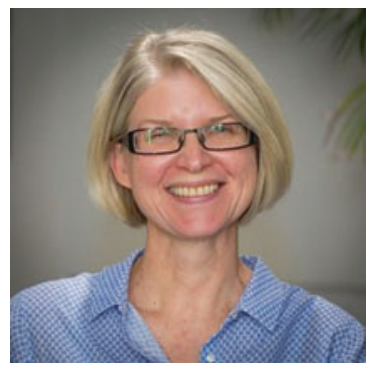

Catherine Haslam School of Psychology, The University of Queensland, Queensland, Australia

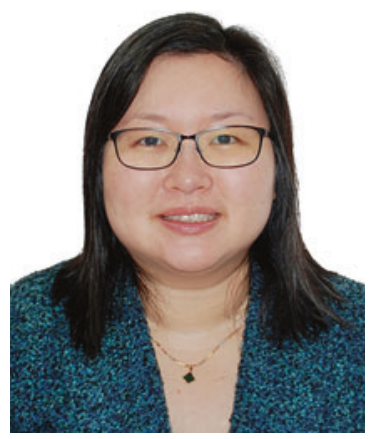

Helene Fung Chinese University of Hong Kong, Shatin, Hong Kong

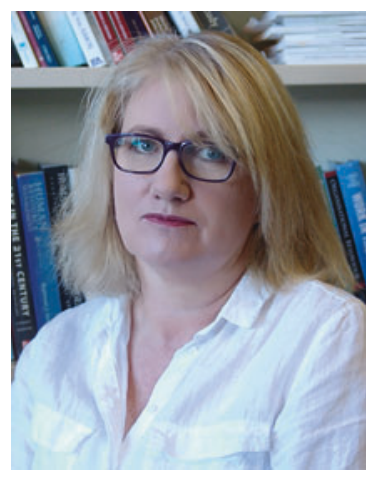

Fiona Alpass School of Psychology, Massey University, Palmerston North, New Zealand 


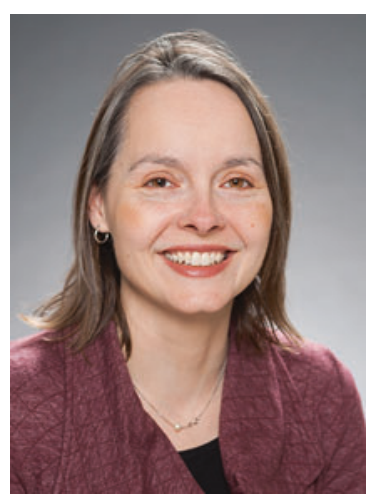

Sherry Ann Beaudreau Psychiatry Service, Sierra Pacific Mental Illness Research Education and Clinical Center (MIRECC), VA Palo Alto Health Care System, Palo Alto, CA, USA

Department of Psychiatry and Behavioral Sciences, Stanford University School of Medicine, Stanford, CA, USA

School of Psychology, University of Queensland, Brisbane, Australia

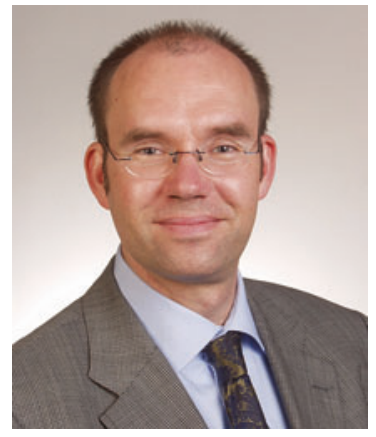

Matthias Kliegel Department of Psychology, University of Geneva, Geneva, Switzerland

Center for the Interdisciplinary Study of Gerontology and Vulnerability, University of Geneva, Geneva, Switzerland

Swiss National Center of Competences in Research LIVES-Overcoming vulnerability: life course perspectives, University of Lausanne Géopolis building, Lausanne, Switzerland 


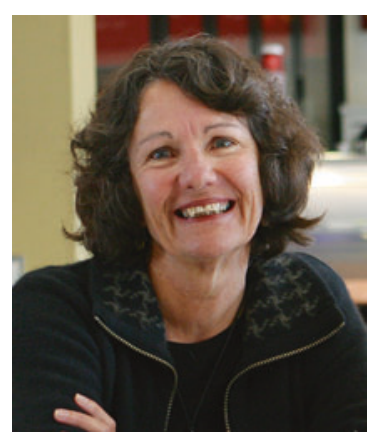

Chris Stephens School of Psychology, Massey University, Palmerston North, New Zealand

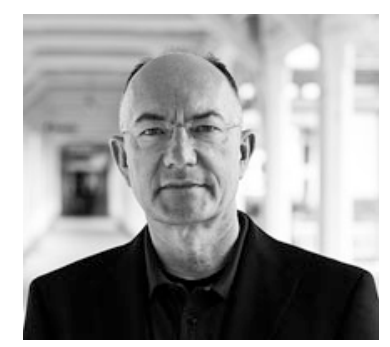

Hans-Werner Wahl Department of Psychological Aging Research, University of Heidelberg, Hauptstrasse, Heidelberg, Germany

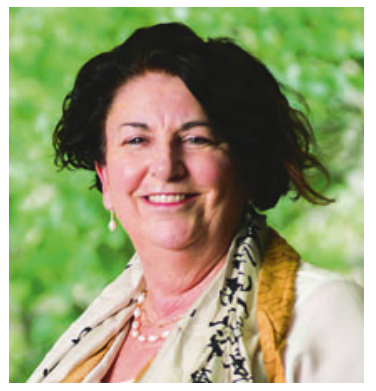

Colette Browning RDNS Institute, Victoria, Australia 


\section{Contributors}

Marja Aartsen NOVA Norwegian Social Research, Oslo and Akershus University College, Oslo, Norway

Phillip L. Ackerman School of Psychology, Georgia Institute of Technology, Atlanta, GA, USA

Stéphane Adam Psychology of Aging Unit, University of Liège, Liège, Belgium

Janne Adolf Max Planck Institute for Human Development, Berlin, Germany

Rosa Marina Afonso Department of Psychology and Education, University of Beira Interior, Covilhã, Portugal

UNIFAI-ICBAS and CINTESIS, University of Porto, Porto, Portugal

Kozma Ahacic Centre for Epidemiology and Community Medicine, Health Care Services, Stockholm County Council, Stockholm, Sweden

Department of Public Health Sciences, Karolinska Institutet, Stockholm, Sweden

Andrew J. Ahrendt University of Nevada, Reno, NV, USA

Julia Alber Center for Health Behavior Research, Perelman School of Medicine, University of Pennsylvania, Philadelphia, PA, USA

Carolyn Aldwin Center for Healthy Aging Research, Oregon State University, Corvallis, OR, USA

Jason C. Allaire Department of Psychology, North Carolina State University, Raleigh-Durham, NC, USA

Mathias Allemand Department of Psychology and University Research Priority Program "Dynamics of Healthy Aging," University of Zurich, Zurich, Switzerland

Joanne Allen School of Psychology, Massey University, Palmerston North, New Zealand

Rebecca S. Allen The University of Alabama, Tuscaloosa, AL, USA

Philip A. Allen Adult Development and Aging Psychology, The University of Akron, Akron, OH, USA 
Fiona Alpass School of Psychology, Massey University, Palmerston North, New Zealand

Lori J. P. Altmann Department of Speech, Language, and Hearing Sciences, University of Florida, Gainesville, FL, USA

Karen Andersen-Ranberg Epidemiology, Biostatistics and Biodemography, Institute of Public Health, University of Southern Denmark, Odense C, Denmark

Kaarin J. Anstey Centre for Research on Ageing Health and Wellbeing, Research School of Population Health, The Australian National University, Canberra, ACT, Australia

Toni C. Antonucci University of Michigan, Ann Arbor, MI, USA

Ivan Aprahamian University of São Paulo, São Paulo, SP, Brazil

Jundiaí Faculty of Medicine, Jundiaí, Brazil

Yasumichi Arai Center for Supercentenarian Research, Keio University School of Medicine, Tokyo, Japan

Lia Araújo UNIFAI and CINTESIS, ICBAS - University of Porto, Porto, Portugal

Portugal and Polytechnic Institute, ESEV and CI\&DETS, Viseu, Portugal

Neal M. Ashkanasy UQ Business School, The University of Queensland, Brisbane, QLD, Australia

Martin Asperholm Division of Psychology, Department of Clinical Neuroscience, Karolinska Instituet, Stockholm, Sweden

Catherine R. Ayers Department of Psychiatry, University of California, San Diego School of Medicine, San Diego, CA, USA

Research Service, VA San Diego Healthcare System, San Diego, CA, USA

Christian Bakker Department of Primary and Community Care: Center for Family Medicine, Geriatric Care and Public Health, Radboud University Medical Center, Nijmegen, The Netherlands

Florence, Mariahoeve, Center for Specialized Care in Young-Onset Dementia, Den Haag, The Netherlands

Radboud Alzheimer Center Nijmegen, Radboud University Medical Center, Nijmegen, The Netherlands

P. Matthijs Bal School of Management, University of Bath, Bath, UK

Andrés Losada Baltar Department of Psychology, Universidad Rey Juan Carlos, Madrid, Spain

James Banks Institute for Fiscal Studies, London, UK

School of Social Sciences, University of Manchester, Manchester, UK 
Fiona Kate Barlow School of Applied Psychology and Menzies Health Institute Queensland, Griffith University, Brisbane, QLD, Australia

Magdalena Bathen Kassel, Germany

Sarah Bauermeister School of Psychology, Faculty of Medicine and Health, University of Leeds, Leeds, UK

Christine Beanland Royal District Nursing Service (RDNS) Institute, Melbourne, VIC, Australia

Sherry A. Beaudreau Department of Psychiatry and Behavioral Sciences, Stanford University School of Medicine, Stanford, CA, USA

Sierra Pacific Mental Illness Research Education and Clinical Center, VA Palo Alto Health Care System, Palo Alto, CA, USA

School of Psychology, The University of Queensland, Brisbane, QLD, Australia

Terry A. Beehr Central Michigan University, Mount Pleasant, MI, USA

Margaret E. Beier Department of Psychology, Rice University, Houston, TX, USA

Raoul Bell Heinrich Heine University Düsseldorf, Düsseldorf, Germany

Sylvie Belleville Psychology Department, Research Centre, Institut Universitaire de Gériatrie de Montréal, Montréal, QC, Canada

Vern L. Bengtson School of Social Work and Edward R. Roybal Institute on Aging, University of Southern California, Los Angeles, CA, USA

Kate M. Bennett Department of Psychological Sciences, University of Liverpool, Liverpool, UK

Karianne Berg Norwegian University of Science and Technology, Trondheim, Norway

Marilena Bertolino Department of Psychology, University de Nice Sophia Antipolis, Nice, France

Maxime Bertoux Norwich Medical School, University of East Anglia, Norfolk, UK

Department of Clinical Neurosciences, University of Cambridge, Cambridge, UK

Sunil S. Bhar Department of Psychological Sciences, Swinburne University of Technology H99, Hawthorn, VIC, Australia

Allison A. M. Bielak Department of Human Development and Family Studies, Colorado State University, Fort Collins, CO, USA

Simon Biggs School of Social and Political Sciences, University of Melbourne, Melbourne, VIC, Australia

Magdalena Bathen: deceased. 
Erin D. Bigler Department of Psychology and Neuroscience Center, Brigham Young University, Provo, UT, USA

Department of Psychiatry, University of Utah, Salt Lake City, UT, USA

Kira S. Birditt Institute for Social Research, University of Michigan, Ann Arbor, MI, USA

Alex J. Bishop Human Development and Family Science Department, Oklahoma State University, Stillwater, OK, USA

Patrizia S. Bisiacchi Department of General Psychology, University of Padova, Padova, Italy

Pär Bjälkebring Department of Psychology, University of Gothenburg, Gothenburg, Sweden

Kathrin Boerner Department of Gerontology, John W. McCormack Graduate School of Policy and Global Studies, University of Massachusetts Boston, Boston, MA, USA

Walter R. Boot Institute for Successful Longevity, Department of Psychology, Florida State University, Tallahassee, FL, USA

Sarah Borish University of California, San Francisco, San Francisco, CA, USA

Axel Börsch-Supan Munich Center for the Economics of Aging, MaxPlanck-Institute for Social Law and Social Policy, Munich, Germany

Tom Borza Centre for Old Age Psychiatric Research, Innlandet Hospital Trust, Oslo, Norway

Nicholas T. Bott Sierra Pacific Mental Illness Research, Education, and Clinical Centers (MIRECC), VA Palo Alto Health Care System, Palo Alto, CA, USA

Pacific Graduate School of Psychology-Stanford PsyD Consortium, Stanford, CA, USA

Catherine E. Bowen Wittgenstein Centre for Demography and Global Human Capital (IIASA, VID/ÖAW, WU), Vienna Institute of Demography/ Austrian Academy of Sciences, Vienna, Austria

S. K. Bradshaw Heart of England Foundation Trust, Birmingham, UK

Caitlin Brandenburg The University of Queensland, St Lucia, Brisbane, QLD, Australia

Daniela Brandão UNIFAI and CINTESIS, ICBAS - University of Porto, Porto, Portugal

Jochen Brandtstädter Department of Psychology, University of Trier, Trier, Germany

Tina Braun Life-Span Developmental Psychology Laboratory, University of Leipzig, Leipzig, Germany 
Mary Breheny School of Public Health, Massey University, Palmerston North, New Zealand

Dawn Brooker University of Worcester Association for Dementia Studies, Institute of Health and Society, University of Worcester, Worcester, UK

Allyson Brothers Department of Human Development and Family Studies, Colorado State University, Fort Collins, CO, USA

Colette J. Browning Royal District Nursing Service, St Kilda, VIC, Australia

International Primary Health Care Research Institute, Shenzhen, China

Monash University, Melbourne, VIC, Australia

Halina Bruce Department of Psychology, Center for Research in Human Development, Concordia University, Montréal, QC, Canada

Hannah Brunet University of California, San Francisco, San Francisco, CA, USA

Axel Buchner Heinrich Heine University Düsseldorf, Düsseldorf, Germany

Romola S. Bucks School of Psychology, University of Western Australia, Crawley, WA, Australia

Gina Bufton School of Psychology, J. S. Coon Building, MC0170, Georgia Institute of Technology, Atlanta, GA, USA

Adam Bulley School of Psychology, The University of Queensland, St Lucia, QLD, Australia

David Bunce School of Psychology, Faculty of Medicine and Health, University of Leeds, Leeds, UK

Céline N. Bürki University Center for Medicine of Aging, Felix PlatterHospital, Basel, Switzerland

Department of Radiology, University of Basel Hospital, Basel, Switzerland

Anne Burmeister Leuphana University of Lüneburg, Lüneburg, Germany

Katherine Burn Department of Medicine - Royal Melbourne Hospital, The University of Melbourne, Parkville, VIC, Australia

Richard A. Burns Centre for Research on Ageing Health and Wellbeing, Research School of Population Health, The Australian National University, Canberra, ACT, Australia

Nicola W. Burton School of Human Movement and Nutrition Sciences, The University of Queensland, St Lucia, Brisbane, QLD, Australia

Alissa M. Butts Mayo Clinic, Rochester, MN, USA

Department of Psychiatry and Psychology, Division of Neurocognitive Disorders, Mayo Clinic, Rochester, MN, USA 
Lisa Calvano West Chester University of Pennsylvania, West Chester, PA, USA

Julieta Camino Faculty of Medicine and Health Sciences, University of East Anglia, Norwich, UK

Katherine Campbell Clinical Psychologist, Department of Psychiatry, The University of Melbourne, Parkville, VIC, Australia

M. Teresa Cardador School of Labor and Employment Relations, University of Illinois, Urbana-Champaign, IL, USA

Keisha D. Carden The University of Alabama, Tuscaloosa, AL, USA

Brian D. Carpenter Department of Psychology, Washington University, St. Louis, MO, USA

Laura L. Carstensen Department of Psychology, Stanford University, Stanford, CA, USA

Maria Teresa Carthery-Goulart Center of Mathematics, Computing and Cognition (CMCC), Federal University of ABC (UFABC), São Bernardo do Campo, São Paulo, Brazil

Cognitive and Behavioral Neurology Unit, Hospital das Clínicas, School of Medicine, University of São Paulo (HCFMUSP), São Paulo, São Paulo, Brazil

Lindsey A. Cary Department of Psychology, University of Toronto, Toronto, ON, Canada

Erin L. Cassidy-Eagle Research and Evaluation, ETR, Scotts Valley, CA, USA

Department of Psychiatry and Behavioral Sciences, Stanford University School of Medicine, Stanford, CA, USA

Casey Cavanagh Department of Psychology, West Virginia University, Morgantown, WV, USA

Jane H. Cerhan Department of Psychiatry and Psychology, Division of Neurocognitive Disorders, Mayo Clinic, Rochester, MN, USA

Eric Cerino School of Social and Behavioral Health Sciences, College of Public Health and Human Sciences, Oregon State University, Corvallis, OR, USA

Veronique S. Chachay School of Human Movement and Nutrition Sciences, School of Medicine, The University of Queensland, Brisbane, QLD, Australia

Faculty of Health and Behavioural Sciences, The University of Queensland, Brisbane, QLD, Australia

Dorey S. Chaffee Department of Psychology, Colorado State University, Fort Collins, CO, USA

Michael C. H. Chan Department of Psychology, Chinese University of Hong Kong, Hong Kong, China 
Anna Chapman RDNS Institute, Melbourne, VIC, Australia

School of Primary Health Care, Monash University, Melbourne, VIC, Australia

Neena L. Chappell Centre on Aging and Department of Sociology, University of Victoria, Victoria, BC, Canada

Susan T. Charles Department of Psychology and Social Behavior, University of California, Irvine, CA, USA

Neil Charness Institute for Successful Longevity, Department of Psychology, Florida State University, Tallahassee, FL, USA

Alison L. Chasteen Department of Psychology, University of Toronto, Toronto, ON, Canada

Xinxin Chen Institute of Social Science Survey, Peking University, Beijing, China

Sheung-Tak Cheng Department of Health and Physical Education, The Education University of Hong Kong, Hong Kong, China

Department of Clinical Psychology, Norwich Medical School, University of East Anglia, Norwich, UK

Monique M. Cherrier Department of Psychiatry and Behavioral Sciences, University of Washington School of Medicine, Seattle, WA, USA

Karen Siu-Lan Cheung Sau Po Centre on Ageing and Department of Social Work and Social Administration, The University of Hong Kong, Hong Kong, China

Adrienne K. Chong University of Nevada, Reno, NV, USA

Kysa M. Christie Boston VA Healthcare System, Boston, MA, USA

Christina Chrysohoou 1st Cardiology Clinic University of Athens, Athens, Greece

Research Institute for Longevity and Prevention of Geriatric Diseases, Athens, Greece

Lindy Clemson Ageing, Work and Health Research Unit, Faculty of Health Sciences, The University of Sydney, Lidcombe, NSW, Australia

Jeanette N. Cleveland Department of Psychology, College of Natural Sciences, Colorado State University, Fort Collins, CO, USA

Simon Cloutier Psychology Department, Research Centre, Institut Universitaire de Gériatrie de Montréal, Montréal, QC, Canada

Giorgia Cona Department of Neuroscience, University of Padova, Padova, Italy

Casey Conaboy Palo Alto University, Palo Alto, CA, USA

Catherine Connaughton Institute for Health and Ageing, Australian Catholic University, Melbourne, VIC, Australia 
Kaitrin Conniff Palo Alto University, Palo Alto, CA, USA

Veterans Affairs Palo Alto Health Care System, Palo Alto, CA, USA

Sarah E. Cook Duke University, Durham, NC, USA

Joel Cooper Princeton University, Princeton, NJ, USA

Ashley D. Cooper Central Michigan University, Mount Pleasant, MI, USA

Laura Coopersmith Palo Alto University, Palo Alto, CA, USA

Nick Corriveau-Lecavalier Psychology Department, Research Centre, Institut Universitaire de Gériatrie de Montréal, Montréal, QC, Canada

John Crawford Centre for Healthy Brain Ageing, University of New South Wales, Sydney, NSW, Australia

Dimity A. Crisp Faculty of Health, University of Canberra, Canberra, ACT, Australia

Tegan Cruwys School of Psychology, The University of Queensland, Brisbane, QLD, Australia

Robert A. Cummins Deakin University, Melbourne, VIC, Australia

Sara J. Czaja University of Miami Miller School of Medicine, Miami, FL, USA

Catriona Daly Centre for Healthy Brain Ageing, University of New South Wales, Sydney, NSW, Australia

Marleen Damman Netherlands Interdisciplinary Demographic Institute (NIDI-KNAW), The Hague, The Netherlands

University Medical Center Groningen, University of Groningen, Groningen, The Netherlands

Tanya Dash Centre de recherche, Institut universitaire de gériatrie de Montréal, Montréal, QC, Canada

École d'orthophonie et d'audiologie, Faculté de médecine, Université de Montréal, QC, Canada

Judith Davey Institute for Governance and Policy Studies, Victoria University of Wellington, Wellington, New Zealand

Danielle K. Davis University of Florida, Gainesville, FL, USA

Liesbeth De Donder Vrije Universiteit Brussel/University College Ghent, Brussels, Belgium

Kiki M. M. De Jonge University of Groningen, Groningen, The Netherlands

Margarida Pedroso de Lima Faculty of Psychology and Educational Sciences, University of Coimbra, Coimbra, Portugal

Jonas Jardim de Paula Faculdade de Ciências Médicas de Minas Gerais, Intituto Nacional de Ciência e Tecnologia em Medicina Molecular, Federal University of Minas Gerais, Belo Horizonte, Minas Gerais, Brazil 
Ans De Vos Antwerp Management School, Antwerp, Belgium

University of Antwerp, Antwerp, Belgium

Marjolein E. de Vugt School for Mental Health and Neuroscience, Alzheimer Center Limburg, Maastricht University Medical Center, Maastricht, The Netherlands

Nico De Witte Vrije Universiteit Brussel/University College Ghent, Brussels, Belgium

University College, Ghent, Belgium

Christina Degen Section of Geriatric Psychiatry, Heidelberg University, Heidelberg, Germany

Serhiy Dekhtyar Division of Psychology, Department of Clinical Neuroscience, Karolinska Instituet, Stockholm, Sweden

Marguerite DeLiema Stanford Center on Longevity, Stanford University, Stanford, CA, USA

Julia A. M. Delius Center for Lifespan Psychology, Max Planck Institute for Human Development, Berlin, Germany

Jürgen Deller Institute of Strategic HR Management Research and Development (SMARD), Leuphana University of Lüneburg, Lüneburg, Germany

Natalie L. Denburg Department of Neurology, University of Iowa Carver College of Medicine, Iowa, IA, USA

Christian J. Lalive d'Epinay Faculty of Sciences of the Society, CIGEV, University of Geneva, Geneva, Switzerland

Donatienne Desmette Institute of Research in Psychological Sciences, Université catholique de Louvain, Louvain-la-Neuve, Belgium

Manfred Diehl Department of Human Development and Family Studies, Colorado State University, Fort Collins, CO, USA

Deborah A. DiGilio American Psychological Association, Washington, DC, USA

Josie Dixon London School of Economics and Political Science, London, UK

Friederike Doerwald Department of Psychology, University of Groningen, Groningen, The Netherlands

Marisa E. Domino Gillings School of Global Public Health, University of North Carolina at Chapel Hill, Chapel Hill, NC, USA

William H. Dow School of Public Health, University of California, Berkeley, CA, USA

Colleen Doyle Australian Catholic University and Villa Maria Catholic Homes, Melbourne, VIC, Australia

National Ageing Research Institute, Melbourne, VIC, Australia 
Mary E. Dozier San Diego State University/University of California, San Diego Joint Doctoral Program in Clinical Psychology, San Diego, CA, USA Research Service, VA San Diego Healthcare System, San Diego, CA, USA

Brian Draper School of Psychiatry, University of NSW, Sydney, NSW, Australia

Academic Department of Old Age Psychiatry, Prince of Wales Hospital, Randwick, NSW, Australia

Natália Duarte UNIFAI and CINTESIS, ICBAS - University of Porto, Porto, Portugal

Patrick Dulin Department of Psychology, University of Alaska, Anchorage, AK, USA

Sandra Düzel Center for Lifespan Psychology, Max Planck Institute for Human Development, Berlin, Germany

Kaitlyn Dykes Sidney Kimmel Medical College, Thomas Jefferson University, Philadelphia, PA, USA

Catherine Earl Federation Business School, Federation University Australia, Churchill, VIC, Australia

Joanne K. Earl Flinders School of Business, Flinders University, Adelaide, SA, Australia

Barry Edelstein Department of Psychology, West Virginia University, Morgantown, WV, USA

Rohan A. Elliott Monash University Centre for Medicine Use and Safety, Melbourne, VIC, Australia

Austin Health Pharmacy Department, Melbourne, VIC, Australia

Michelle L. Ellis School of Aging Studies, University of South Florida, Tampa, FL, USA

Diane Elmore Policy Program, UCLA-Duke University National Center for Child Traumatic Stress, Washington, DC, USA

Tammy English Washington University in St. Louis, St. Louis, MO, USA

Alexandra Ernst LEAD CNRS UMR 5022, Université de Bourgogne, Dijon, France

University of Burgundy, Dijon, France

J. Kaci Fairchild Sierra Pacific Mental Illness Research Education and Clinical Center, VA Palo Alto Health Care System, Stanford University School of Medicine, Palo Alto, CA, USA

Yang Fang Department of Psychology, The Chinese University of Hong Kong, Hong Kong, China 
Thomas J. Farrer Department of Psychiatry and Behavioral Sciences, Duke University Medical Center, Durham, NC, USA

Ulrike Fasbender Department of Business and Management, Oxford Brookes University, Oxford, UK

Joanne Feeney The Irish Longitudinal Study on Ageing, Department of Medical Gerontology, Trinity College Dublin, Dublin, Ireland

Centre for Public Health, Queen's University Belfast, Belfast, Northern Ireland, United Kingdom

Lei Feng Department of Psychological Medicine, Yong Loo Lin School of Medicine, National University of Singapore, Singapore, Singapore

Qiushi Feng Department of Sociology, National University of Singapore, Singapore, Singapore

Daniela Figueiredo School of Health Sciences, University of Aveiro, Aveiro, Portugal

Center for Health Technology and Services Research (CINTESIS.UA), Aveiro, Portugal

Karen Fingerman Human Development and Family Sciences, Population Research Center, The College of Liberal Arts, University of Texas-Austin, Austin, TX, USA

Gwenith G. Fisher Department of Psychology, Colorado State University, Fort Collins, CO, USA

Jane E. Fisher Department of Psychology/298, University of Nevada, Reno, NV, USA

Amy Fiske Department of Psychology, West Virginia University, Morgantown, WV, USA

Emma Flanagan Norwich Medical School, University of East Anglia, Norfolk, UK

Matt Flynn Centre for Research into the Older Workforce (CROW), Newcastle University, Newcastle upon Tyne, UK

Kitty-Rose Foley Department of Developmental Disability Neuropsychiatry, School of Psychiatry, University of New South Wales, Sydney, NSW, Australia

Cooperative Research Centre for Living with Autism (Autism CRC), Long Pocket, Brisbane, QLD, Australia

Evgenia Folts Department of Pediatrics, University of Iowa Carver College of Medicine, Iowa, IA, USA

Simon Forstmeier Faculty II - Department of Education Studies and Psychology, Developmental Psychology, University of Siegen, Siegen, Germany 
Franco Fraccaroli Department of Psychology and Cognitive Science, University of Trento, Trento, Italy

Susan Freiberg Institute for Work and Health of the German Social Accident Insurance, Dresden, Germany

Alexandra M. Freund Department of Psychology, University of Zurich, Zurich, Switzerland

University Research Priority Program Dynamics of Healthy Aging, University of Zurich, Zurich, Switzerland

Helene H. Fung Department of Psychology, The Chinese University of Hong Kong, Hong Kong, China

Rebecca Funken Institute of Strategic HR Management, Leuphana University of Lüneburg, Lüneburg, Germany

Trude Furunes Norwegian School of Hotel Management, University of Stavanger, Stavanger, Norway

Alyssa A. Gamlado School of Aging Studies, University of South Florida, Tampa, FL, USA

Christina Garrison-Diehn Geriatric Research, Education, and Clinical Center, VA Palo Alto Health Care System, Palo Alto, CA, USA

Department of Psychiatry and Behavioral Science, Stanford University School of Medicine, Stanford, CA, USA

Daniela Garten Department of Politics and Public Administration, University of Konstanz, Konstanz, Germany

Joseph E. Gaugler School of Nursing, University of Minnesota, Minneapolis, MN, USA

Zvi D. Gellis School of Social Policy and Practice, Center for Mental Health and Aging, University of Pennsylvania, Philadelphia, PA, USA

Debby L. Gerritsen Department of Primary and Community Care: Center for Family Medicine, Geriatric Care and Public Health, Radboud University Medical Center, Nijmegen, The Netherlands

Radboud Alzheimer Center Nijmegen, Radboud University Medical Center, Nijmegen, The Netherlands

Denis Gerstorf Institute of Psychology, Humboldt University, Berlin, Germany

Michael M. Gielnik Institute of Strategic HR Management, Leuphana University of Lüneburg, Lüneburg, Germany

Jacqueline M. Gilberto Department of Psychology, Rice University, Houston, TX, USA 
Karen Glanz Perelman School of Medicine, University of Pennsylvania, Philadelphia, PA, USA

Lisa H. Glassman VA San Diego Healthcare System, San Diego, CA, USA University of California, San Diego, San Diego, CA, USA

Judith Glück Department of Psychology, Alpen-Adria-Universität Klagenfurt, Klagenfurt, Austria

Rowena Gomez Pacific Graduate School of Psychology, Palo Alto University, Palo Alto, CA, USA

Yasuyuki Gondo Department of Clinical Thanatology and Geriatric Behavioral Science, Osaka University Graduate School of Human Sciences, Suita, Japan

Xianmin Gong Department of Psychology, The Chinese University of Hong Kong, Hong Kong, China

B. Heath Gordon Mental Health, G.V. (Sonny) Montgomery Veterans Affairs Medical Center, Jackson, MS, USA

Christine E. Gould Department of Psychiatry and Behavioral Sciences, Stanford University School of Medicine, Stanford, CA, USA

Geriatric Research, Education, and Clinical Center (GRECC), VA Palo Alto Health Care System, Palo Alto, CA, USA

Alan J. Gow Department of Psychology, Heriot-Watt University, Edinburgh, UK

Jeffrey J. Gregg Durham Veterans Affairs Medical Center, Durham, NC, USA

Julie Gretler Palo Alto University, Palo Alto, CA, USA

Werner Greve Hildesheim University, Hildesheim, Germany

Catherine Grotz Psychology of Aging Unit, University of Liège, Liège, Belgium

Danan Gu United Nations Population Division, New York, NY, USA

Angela H. Gutchess Brandeis University, Waltham, MA, USA

Thomas Hadjistavropoulos Department of Psychology and Centre on Aging and Health, University of Regina, Regina, SK, Canada

Joachim Hallmayer Department of Psychiatry and Behavioral Sciences, Stanford University School of Medicine, Stanford University, Stanford, CA, USA

Sierra Pacific Mental Illness Research Education and Clinical Center (MIRECC), VA Palo Alto Health Care System, Palo Alto, CA, USA 
Madison E. Hanscom Department of Psychology, College of Natural Sciences, Colorado State University, Fort Collins, CO, USA

Nathan Hantke Sierra Pacific Mental Illness Research, Education, and Clinical Centers (MIRECC), VA Palo Alto Health Care System, Palo Alto, CA, USA

Department of Psychiatry and Behavioral Sciences, Stanford University School of Medicine, Stanford, CA, USA

Lynn Hasher Department of Psychology, University of Toronto, Toronto, ON, USA

Rotman Research Institute, Baycrest, Toronto, ON, USA

Hideki Hashimoto Department of Health and Social Behavior, The University of Tokyo School of Public Health, Bunkyo, Tokyo, Japan

S. Alexander Haslam School of Psychology, The University of Queensland, Brisbane, QLD, Australia

Catherine Haslam School of Psychology, The University of Queensland, Brisbane, QLD, Australia

Louise C. Hawkley Academic Research Centers, NORC at the University of Chicago, Chicago, IL, USA

Tyler Haydell Stanford University, Stanford, CA, USA

Becky I. Haynes School of Psychology, Faculty of Medicine and Health, University of Leeds, Leeds, UK

Jutta Heckhausen Department of Psychology and Social Behavior, School of Social Ecology, University of California, Irvine, CA, USA

Chyrisse Heine College of Science Health and Engineering, Department of Community and Clinical Allied Health, School of Allied Health, La Trobe University, Melbourne, VIC, Australia

Edward Helmes Department of Psychology, James Cook University, Townsville, QLD, Australia

Gert-Jan Hendriks Pro Persona Institute for Integrated Mental Health Care, Centre for Anxiety Disorders “Overwaal," Nijmegen, The Netherlands

Radboud University Nijmegen, Behavioural Science Institute, Nijmegen, The Netherlands

Radboud University Medical Centre, Department of Psychiatry, Nijmegen, The Netherlands

Julie D. Henry School of Psychology, The University of Queensland, Brisbane, QLD, Australia

Noreen Heraty Kemmy Business School, University of Limerick, Limerick, Ireland 
Agneta Herlitz Division of Psychology, Department of Clinical Neuroscience, Karolinska Instituet, Stockholm, Sweden

Guido Hertel Department of Psychology, University of Münster, Münster, Germany

Christopher Hertzog School of Psychology, Georgia Institute of Technology, Atlanta, GA, USA

Thomas M. Hess Department of Psychology, North Carolina State University, Raleigh, NC, USA

Stephanie Hicks Psychology Department, Fordham University, Bronx, NY, USA

Patrick L. Hill Department of Psychology, Carleton University, Ottawa, ON, Canada

Thomas Hinault Aix-Marseille Université and CNRS, Marseille, France

Gregory A. Hinrichsen Department of Geriatrics and Palliative Medicine, Icahn School of Medicine at Mount Sinai, New York, NY, USA

Courtney von Hippel School of Psychology, The University of Queensland, Brisbane, QLD, Australia

William von Hippel School of Psychology, The University of Queensland, Brisbane, QLD, Australia

Nobuyoshi Hirose Center for Supercentenarian Research, Keio University School of Medicine, Tokyo, Japan

Andreas Hirschi Institute of Psychology, University of Bern, Bern, Switzerland

Rayna Hirst Palo Alto University, Palo Alto, CA, USA

Henry C. Y. Ho School of Public Health, University of Hong Kong, Hong Kong, China

Lieve Hoeyberghs University College, Ghent, Belgium

Joanna Hong Department of Psychology and Social Behavior, University of California, Irvine, CA, USA

Karen Hooker School of Social and Behavioral Health Sciences, College of Public Health and Human Sciences, Oregon State University, Corvallis, OR, USA

Christiane A. Hoppmann Department of Psychology, University of British Columbia, Vancouver, BC, Canada

Michael Hornberger Norwich Medical School, University of East Anglia, Norfolk, UK

Lena-Alyeska M. Huebner Department of Psychology, College of Natural Sciences, Colorado State University, Fort Collins, CO, USA 
J. W. Terri Huh VA Palo Alto Health Care System, Palo Alto, CA, USA

Stanford University School of Medicine, Stanford, CA, USA

Martijn Huisman NOVA Norwegian Social Research, Oslo and Akershus University College, Oslo, Norway

EMGO Institute for Health and Care Research, VU University Medical Center, Amsterdam, The Netherlands

Department of Epidemiology and Biostatistics, VU University Medical Center, Amsterdam, The Netherlands

Department of Psychiatry, VU University Medical Center, Amsterdam, The Netherlands

Mary Lee Hummert Communication Studies Department, University of Kansas, Lawrence, KS, USA

Ye In (Jane) Hwang Department of Developmental Disability Neuropsychiatry, School of Psychiatry, University of New South Wales, Sydney, NSW, Australia

Cooperative Research Centre for Living with Autism (Autism CRC), Long Pocket, Brisbane, QLD, Australia

Maria Iankilevitch Department of Psychology, University of Toronto, Toronto, ON, Canada

Kazunori Ikebe Department of Prosthodontics, Gerodontology and Oral Rehabilitation, Osaka University Graduate School of Dentistry, Suita, Japan

Hiroki Inagaki Research Team for Promoting Independence of the Elderly, Tokyo Metropolitan Institute of Gerontology, Tokyo, Japan

Jennifer Inauen Department of Psychology, Columbia University, New York, NY, USA

Derek M. Isaacowitz Department of Psychology, Northeastern University, Boston, MA, USA

Yoshiko Lily Ishioka Tokyo Metropolitan Institute of Gerontology, Tokyo, Japan

Graduate School of Science and Technology, Keio University, Yokohama, Japan

Tatsuro Ishizaki Tokyo Metropolitan Geriatric Hospital and Institute of Gerontology, Tokyo, Japan

Shelly L. Jackson Institute of Law, Psychiatry and Public Policy, University of Virginia, Charlottesville, VA, USA

Lori E. James Psychology Department, University of Colorado, Colorado Springs, CO, USA 
Soong-Nang Jang Red Cross College of Nursing, Chung-Ang University, Seoul, South Korea

Jolanda Jetten School of Psychology, The University of Queensland, Brisbane, QLD, Australia

Garima Jhingon Pacific Graduate School of Psychology, Palo Alto University, Palo Alto, CA, USA

Da Jiang Department of Psychology, Chinese University of Hong Kong, Hong Kong, China

Yves Joanette Centre de recherche, Institut universitaire de gériatrie de Montréal, Montréal, QC, Canada

Boo Johansson Department of Psychology, University of Gothenburg, Gothenburg, Sweden

Mary Ann Johnson Department of Foods and Nutrition, University of Georgia, Athens, GA, USA

Claire S. Johnston Institute of Psychology, University of Bern, Bern, Switzerland

Daniela S. Jopp Institute of Psychology, University of Lausanne, Lausanne, Switzerland

Swiss Centre of Competence in Research LIVES, Overcoming Vulnerability: Life Course Perspectives, Lausanne, Switzerland

Josh Jordan Department of Psychiatry and Behavioral Sciences, Stanford University School of Medicine, Stanford University, Stanford, CA, USA

California School of Professional Psychology, Alliant International University, Alhambra, CA, USA

Bruce Judd Australian School of Architecture and Design, Built Environment, University of New South Wales, Sydney, NSW, Australia

Seojung Jung Fordham University, New York, NY, USA

Franziska Jungmann Institute for Work, Organizational and Social Psychology, University of Technology, Dresden, Dresden, Germany

Elise K. Kalokerinos Department of Psychology and Educational Sciences, KU Leuven, Leuven, Flemish Brabant, Belgium

Kei Kamide Department of Health Science and Department of Geriatric Medicine and Nephrology, Osaka University, Graduate School of Medicine, Suita, Japan

Ruth Kanfer School of Psychology, J. S. Coon Building, MC0170, Georgia Institute of Technology, Atlanta, GA, USA

Julia Karbach Goethe-University Frankfurt, Frankfurt, Germany 
Michele J. Karel Mental Health Services, Department of Veterans Affairs Central Office, Washington, DC, USA

Julia E. Kasl-Godley Palo Alto VA Health Care System, Palo Alto, CA, USA

Joseph S. Kay Department of Psychology and Social Behavior, School of Social Ecology, University of California, Irvine, CA, USA

Hal Kendig Australian National University, Canberra, ACT, Australia

Robert F. Kennison Department of Psychology, California State University, Los Angeles, CA, USA

Rose Anne Kenny The Irish Longitudinal Study on Ageing, Department of Medical Gerontology, Trinity College Dublin, Dublin, Ireland

Mercer's Institute for Successful Ageing, St. James' Hospital, Dublin, Ireland

Ngaire Kerse Department of General Practice and Primary Health Care, School of Population Health, The University of Auckland, Auckland, New Zealand

Eva-Marie Kessler MSB Medical School Berlin Hochschule für Gesundheit und MedizinCalandrellistrasse, , Berlin, Germany

Kim M. Kiely Centre for Research on Ageing Health and Wellbeing, Research School of Population Health, The Australian National University, Canberra, ACT, Australia

Douglas C. Kimmel City College, City University of New York, New York, NY, USA

David B. King IRMACS Centre, Simon Fraser University, Burnaby, BC, Canada

Susan Kirkland Departments of Community Health and Epidemiology and Medicine, Dalhousie University, Dalhousie, NS, Canada

Douglas A. Kleiber University of Georgia College of Education, Athens, GA, USA

Matthias Kliegel Department of Psychology, University of Geneva, Geneve 4, Switzerland

Center for Interdisciplinary Study of Gerontology and Vulnerability (CIGEV), University of Geneva, Carouge, Switzerland

Andrzej Klimczuk Warsaw School of Economics, Warsaw, Poland

Michaela Knecht Department of Psychology, University of Zurich, Zurich, Switzerland

University Research Priority Program Dynamics of Healthy Aging, University of Zurich, Zurich, Switzerland 
Jamie E. Knight Department of Psychology, University of Victoria, Victoria, $\mathrm{BC}$, Canada

Dorien Kooij Department of Human Resource Studies, Tilburg University, Tilburg, Netherlands

Raymond T. C. M. Koopmans Department of Primary and Community Care: Center for Family Medicine, Geriatric Care and Public Health, Radboud University Medical Center, Nijmegen, The Netherlands

Radboud Alzheimer Center Nijmegen, Radboud University Medical Center, Nijmegen, The Netherlands

Joachim en Anna, Center for specialized geriatric care, Nijmegen, The Netherlands

Anna E. Kornadt Department of Psychology, Bielefeld University, Bielefeld, Germany

Pavel Kozik Department of Psychology, University of British Columbia, Vancouver, BC, Canada

Abigail Kramer Sierra Pacific Mental Illness Research, Education, and Clinical Centers (MIRECC), VA Palo Alto Health Care System, Palo Alto, CA, USA

Pacific Graduate School of Psychology, Palo Alto University, Palo Alto, CA, USA

Joel Kramer Unviersity of California, San Francisco, San Francisco, CA, USA

Ralf T. Krampe Brain and Cognition, University of Leuven, Leuven, Belgium

Jutta Kray Saarland University, Saarbrücken, Germany

Department of Psychology, Saarland University, Saarbrücken, Saarland, Germany

Kamini Krishnan Mayo Clinic, Rochester, MN, USA

Andreas Kruse Institute of Gerontology, University of Heidelberg, Heidelberg, Germany

Alexis Kuerbis Silberman School of Social Work, Hunter College of the City University of New York, New York, NY, USA

Florian Kunze Department of Politics and Public Administration, University of Konstanz, Konstanz, Germany

Ute Kunzmann Life-Span Developmental Psychology Laboratory, University of Leipzig, Leipzig, Germany

Tanja Kurtz University of Mainz, Mainz, Germany 
Dawn La Palo Alto University/Pacific Graduate School of Psychology, Palo Alto, CA, USA

Sierra Pacific Mental Illness, Research Education and Clinical Centers at the Veterans Affairs Palo Alto Health Care System, Palo Alto, CA, USA

Geoffrey Lane Psychology Service, VA Palo Alto Healthcare System Livermore Division, Livermore, CA, USA

Douglas Warren Lane Geriatrics and Extended Care Service, VA Puget Sound Healthcare System, Seattle, WA, USA

Department of Psychiatry, University of Washington, Seattle, WA, USA

Frieder R. Lang Institute of Psychogerontology, Friedrich-Alexander-University of Erlangen-Nürnberg, Nürnberg, Germany

José Miguel Latorre Postigo Department of Psychology, Faculty of Medicine, University of Castilla-La Mancha, Albacete, Spain

Bobo Hi-Po Lau Faculty of Social Sciences, The University of Hong Kong, Hong Kong, China

Gary D. Laver Psychology and Child Development Department, Cal Poly, San Luis Obispo, CA, USA

Jennifer C. Lay The University of British Columbia, Vancouver, BC, Canada

George Lazaros 1st Cardiology Clinic University of Athens, Athens, Greece

Research Institute for Longevity and Prevention of Geriatric Diseases, Athens, Greece

Malloy-Diniz Leandro Fernandes Department of Mental Health, Instituto Nacional de Ciência e Tecnologia em Medicina Molecular, Federal University of Minas Gerais, Belo Horizonte, Minas Gerais, Brazil

Shinduk Lee Texas A\&M Health Science Center, School of Public Health, College Station, TX, USA

Yunhwan Lee Department of Preventive Medicine and Public Health, Ajou University School of Medicine, Suwon, Republic of Korea

Institute on Aging, Ajou University Medical Center, Suwon, Republic of Korea

Cik Yin Lee Royal District Nursing Service (RDNS) Institute, Melbourne, VIC, Australia

Centre for Medicine Use and Safety, Monash University, Melbourne, VIC, Australia

Patrick Lemaire Aix-Marseille Université and CNRS, Marseille, France

Shu-Chen Li Department of Psychology Chair of Lifespan Developmental Neuroscience, TU Dresden, Dresden, Germany 
Center for Lifespan Psychology, Max Planck Institute for Human Development, Berlin, Germany

Karen Z.H. Li Department of Psychology, Center for Research in Human Development, Concordia University, Montréal, QC, Canada

Tianyuan Li Department of Psychological Studies and Centre for Psychosocial Health, Hong Kong Institute of Education, Tai Po, New Territories, Hong Kong, China

Mei-Ching Lien School of Psychological Science, Oregon State University, Corvallis, OR, USA

Ulman Lindenberger Center for Lifespan Psychology, Max Planck Institute for Human Development, Berlin, Germany

Victoria Liou-Johnson University of California, San Francisco, CA, USA

Sierra Pacific Mental Illness Research Education and Clinical Center, VA Palo Alto Health Care System, Palo Alto, CA, USA

Shuang Liu School of Communication and Arts, The University of Queensland, Brisbane, QLD, Australia

Kimberly M. Livingstone Department of Psychology, Northeastern University, Boston, MA, USA

Vanessa M. Loaiza University of Essex, Colchester, United Kingdom

Ada Lo The University of Queensland, St Lucia, QLD, Australia

Corinna E. Löckenhoff Department of Human Development, Cornell University, Ithaca, NY, USA

Peggy Lockhart Iowa State University, Ames, IA, USA

Alexandra Lopes Institute of Sociology, University of Porto, Porto, Portugal

Andrés Losada-Baltar Department of Psychology, Facultad de Ciencias de la Salud, Universidad Rey Juan Carlos, Madrid, Spain

Katie Louwagie School of Nursing, University of Minnesota, Minneapolis, MN, USA

Martin Lövdén Aging Research Center, Karolinska Institutet and Stockholm University, Stockholm, Sweden

Judy Lowthian School of Public Health and Preventive Medicine, Monash University, Melbourne, VIC, Australia

Minjie Lu Department of Psychology, The Chinese University of Hong Kong, Hong Kong, China

John A. Lucas Department of Psychiatry and Psychology, Mayo Clinic, Jacksonville, FL, USA

Terry Lum The University of Hong Kong, Hong Kong, China 
Angela Lunde Mayo Clinic, Rochester, MN, USA

Mary A. Luszcz Flinders University, Adelaide, SA, Australia

David Madden Brain Imaging and Analysis Center, Duke University Medical Center, Durham, NC, USA

Justin Marcus Ozyegin University, Istanbul, Turkey

Jennifer Margrett Iowa State University, Ames, IA, USA

Rodrigo Mariño Oral Health Cooperative Research Centre, Melbourne Dental School, University of Melbourne, Melbourne, VIC, Australia

María Márquez-González Departament of Biological and Health Psychology, Universidad Autónoma de Madrid, Madrid, Spain

Donel M. Martin Black Dog Institute, School of Psychiatry, University of New South Wales, Sydney, NSW, Australia

Peter Martin Iowa State University, Ames, IA, USA

Yukie Masui Tokyo Metropolitan Geriatric Hospital and Institute of Gerontology, Tokyo, Japan

Rui Mata University of Basel, Basel, Switzerland

Karen A. Mather Centre for Healthy Brain Ageing, University of New South Wales, Sydney, NSW, Australia

Katey Matthews CMIST, School of Social Sciences, University of Manchester, Manchester, UK

Susanne Mayr Heinrich Heine University Düsseldorf, Düsseldorf, Germany University of Passau, Passau, Germany

Marita McCabe Institute for Health and Ageing, Australian Catholic University, Melbourne, VIC, Australia

Jean McCarthy Kemmy Business School, University of Limerick, Limerick, Ireland

Shawn M. McClintock Division of Psychology, Department of Psychiatry, UT Southwestern Medical Center, Dallas, TX, USA

Division of Brain Stimulation and Neurophysiology, Department of Psychiatry and Behavioral Sciences, Duke University School of Medicine, Durham, NC, USA

Michael Thomas McGann School of Social and Political Sciences, University of Melbourne, Melbourne, VIC, Australia

Judy McGregor AUT University, Auckland, New Zealand

Bernard McKenna The University of Queensland, Brisbane, QLD, Australia 
Christopher McLoughlin Federation Business School, Federation University Australia, Churchill, VIC, Australia

Verena H. Menec University of Manitoba, Winnipeg, MB, Canada

Claudia Meyer RDNS Institute, St Kilda, VIC, Australia

Centre for Health Communication and Participation, School of Psychology and Public Health, LaTrobe University, VIC, Australia

Lisa Mieskowski University of Alabama, Tuscaloosa, AL, USA

Victoria Michalowski Department of Psychology, University of British Columbia, Vancouver, BC, Canada

Joany K. Millenaar School for Mental Health and Neuroscience, Alzheimer Center Limburg, Maastricht University Medical Center, Maastricht, The Netherlands

Brent Mills Palo Alto University, Palo Alto, CA, USA

Veterans Affairs Palo Alto Health Care System, Palo Alto, CA, USA

Sierra Pacific Mental Illness Research Education and Clinical Center, VA Palo Alto Health Care System, Palo Alto, CA, USA

Beyon Miloyan Department of Mental Health, Johns Hopkins Bloomberg School of Public Health, Baltimore, MD, USA

Eneida Mioshi Faculty of Medicine and Health Sciences, University of East Anglia, Norwich, UK

Leander K. Mitchell School of Psychology, The University of Queensland, Brisbane, QLD, Australia

Sepideh Modrek School of Medicine, Stanford University, Palo Alto, CA, USA

Scott D. Moffat Georgia Institute of Technology, Atlanta, GA, USA

Darya Moghimi Department of Psychology, University of Groningen, Groningen, The Netherlands

Victor Molinari School of Aging Studies, University of South Florida, Tampa, FL, USA

Terri G. Monk Department of Anesthesiology and Perioperative Medicine, University of Missouri-Columbia, Columbia, MO, USA

Alison A. Moore Division of Geriatric Medicine, David Geffen School of Medicine at UCLA, Los Angeles, CA, USA

Caitlin S. Moore Ryan Dolby Brain Health Center, California Pacific Medical Center Neurosciences Institute, San Francisco, CA, USA

H. C. Moorey Heart of England Foundation Trust, Birmingham, UK 
Lafaiete Guimarães Moreira Universidade Fundação Mineira de Educação e Cultura - FUMEC, Belo Horizonte, Minas Gerais, Brazil

Georgina Moreno Department of Psychology, New York University, New York, NY, USA

Mike Morgan Oral Health Cooperative Research Centre, Melbourne Dental School, University of Melbourne, Melbourne, VIC, Australia

Steven Morrison School of Physical Therapy and Athletic Training, Old Dominion University, Norfolk, VA, USA

Moyra E. Mortby Centre for Research on Ageing, Health and Wellbeing, The Australian National University, Canberra, ACT, Australia

Thomas A. Morton Psychology, College of life and Environmental Sciences, University of Exeter, Exeter, UK

Chris J.A. Moulin LEAD CNRS UMR 5022, Université de Bourgogne, Dijon, France

University of Burgundy, Dijon, France

Christopher Moulin Laboratoire de Psychologie and Neurocognition (LPNC), CNRS-UMR 5105, University Grenoble Alpes, Grenoble, France

Julia Muenchhoff Centre for Healthy Brain Ageing, University of New South Wales, Sydney, NSW, Australia

Andreas Müller Institute for Occupational and Social Medicine, Medical Faculty, Düsseldorf University, Düsseldorf, Germany

Jo Munro Ageing, Work and Health Research Unit, Faculty of Health Sciences, The University of Sydney, Lidcombe, NSW, Australia

Alexa M. Muratore Sydney, NSW, Australia

Marama Muru-Lanning James Henare Research Centre, The University of Auckland, Auckland, New Zealand

Reidar J. Mykletun Molde University College, Molde, Norway

Noemi Nagy Institute of Psychology, University of Bern, Bern, Switzerland

Christopher M. Napolitano Department of Psychology, University of Zurich, Zurich, Switzerland

Paul Nash Centre for Innovative Ageing, Swansea University, Wales, UK

James Nazroo School of Social Sciences, University of Manchester, Manchester, UK

Holly Nelson-Becker Loyola University School of Social Work, Chicago, IL, USA

Karl M. Newell The University of Georgia, Athens, GA, USA

Nicky J. Newton Wilfrid Laurier University, Waterloo, ON, Canada 
MyNhi Nguyen School of Psychology, The University of Queensland, Brisbane, QLD, Australia

Victoria Nieborowska Department of Psychology, Center for Research in Human Development, Concordia University, Montréal, QC, Canada

Jonna Nilsson Aging Research Center, Karolinska Institutet and Stockholm University, Stockholm, Sweden

Ina Nitschke Clinic for Gerodontology and Special Care Dentistry, University of Zurich, Zurich, Switzerland

J. Farley Norman Department of Psychological Sciences, Ogden College of Science and Engineering, Western Kentucky University, Bowling Green, KY, USA

Thomas A. Norton School of Psychology, The University of Queensland, Brisbane, QLD, Australia

Nanna Notthoff Institute of Psychology, Humboldt University, Berlin, Germany

Klaus Oberauer University of Zurich, Zurich, Switzerland

Claire O'Callaghan Behavioral and Clinical Neurosciences Institute, University of Cambridge, Cambridge, UK

Michael P. O'Driscoll School of Psychology, University of Waikato, Hamilton, New Zealand

Ruth O'Hara Department of Psychiatry and Behavioral Sciences, Stanford University School of Medicine, Stanford, CA, USA

Sierra Pacific Mental Illness Research Education and Clinical Center, VA Palo Alto Health Care System, Palo Alto, CA, USA

School of Psychology, The University of Queensland, Brisbane, QLD, Australia

Michelle Olaithe School of Psychology, University of Western Australia, Crawley, WA, Australia

Norm O'Rourke Department of Public Health, Ben-Gurion University of the Negev, Be'er Sheva, Israel

Marcia G. Ory Health Promotion and Community Health Sciences, Texas A\&M Health Science Center and School of Public Health, College Station, TX, USA

Nancy A. Pachana School of Psychology, The University of Queensland, Brisbane, QLD, Australia

Laura E. Paige Brandeis University, Waltham, MA, USA

Nicole E. Pardo Remind Technologies Inc., Houston, TX, USA

Sang Chul Park Department of New Biology, DGIST, Daegu, South Korea 
Stacey L. Parker School of Psychology, The University of Queensland, Brisbane, QLD, Australia

Mario A. Parra Department of Psychology, Centre for Cognitive Ageing and Cognitive Epidemiology, and Human Cognitive Neuroscience, The University of Edinburgh, Edinburgh, UK

Department of Psychology, Heriot-Watt University, Edinburgh, UK

Alzheimer Scotland Dementia Research Centre, The University of Edinburgh, Edinburgh, UK

UDP-INECO Foundation Core on Neuroscience (UIFCoN), Diego Portales University, Santiago, Chile

Constança Paúl UNIFAI and CINTESIS, ICBAS - University of Porto, Porto, Portugal

Yaritza D. Perez-Hooks Princeton University, Princeton, NJ, USA

Giovanni Mario Pes Department of Clinical and Experimental Medicine, University of Sassari, Sassari, Sardinia, Italy

National Institute of Biostructures and Biosystems, University of Sassari, Sassari, Italy

Andrew J. Petkus Department of Psychology, University of Southern California, Los Angeles, CA, USA

Louise H. Phillips University of Aberdeen, Aberdeen, UK

I. Philp Heart of England Foundation Trust, Birmingham, UK

Andrea M. Piccinin Department of Psychology, University of Victoria, Victoria, BC, Canada

Christos Pitsavos 1st Cardiology Clinic University of Athens, Athens, Greece

Research Institute for Longevity and Prevention of Geriatric Diseases, Athens, Greece

Leonard W. Poon University of Georgia, Athens, GA, USA

Lauren E. Popham Greenwald \& Associates, Washington, DC, USA

Michel Poulain IACCHOS Institute of Analysis of Change in Contemporary and Historical Societies, Université catholique de Louvain, Louvain-LaNeuve, Belgium

Estonian Institute for Population Studies, Tallinn University, Tallinn, Estonia

Emma E. Poulsen School of Psychology, The University of Queensland, Brisbane, QLD, Australia

Catherine C. Price Department of Clinical and Health Psychology, University of Florida, Gainesville, FL, USA

Department of Anesthesiology, University of Florida, Gainesville, FL, USA 
Elizabeth C. Price Department of Psychology, West Virginia University, Morgantown, WV, USA

Janice C. Probst Arnold School of Public Health, University of South Carolina, Columbia, SC, USA

Jeffrey Proulx Department of Neurology, School of Medicine, Oregon Health and Science University, Portland, OR, USA

Amy C. Pytlovany Department of Psychology, Portland State University, Portland, OR, USA

Sara Honn Qualls Gerontology Center, University of Colorado, Colorado Springs, Colorado Springs, CO, USA

Patrick Rabbitt Department of Experimental Psychology, University of Oxford, Oxford, UK

Wiebke Rahmlow Department of Psychiatry and Psychotherapy, University of Leipzig, Leipzig, Germany

Parminder Raina Department of Clinical Epidemiology and Biostatistics, McMaster University, Hamilton, ON, Canada

Peter Rammelsberg Department of Prosthodontics, University Hospital Heidelberg, Heidelberg, Germany

G. Kevin Randall Human Sciences Extension and Outreach, Partnerships in Prevention Science Institute, Iowa State University, Ames, IA, USA

Signe Hoei Rasmussen Epidemiology, Biostatistics and Biodemography, Institute of Public Health, University of Southern Denmark, Odense C, Denmark

Philippe Rast Department of Psychology, University of Victoria, Victoria, BC, Canada

Andrew E. Reed Department of Psychology, Stanford University, Stanford, CA, USA

Gwyneth Rees Department of Surgery, Centre for Eye Research Australia, Royal Victorian Eye and Ear Hospital, Ophthalmology, University of Melbourne, Melbourne, VIC, Australia

Laura Simpson Reeves Institute for Social Science Research, The University of Queensland, Brisbane, QLD, Australia

Barbara Resnick University of Maryland School of Nursing, Baltimore, MD, USA

Nancy Reyes Department of Psychology, California State University, Los Angeles, CA, USA

Stephen Rhodes Department of Psychology, Centre for Cognitive Ageing and Cognitive Epidemiology, and Human Cognitive Neuroscience, The University of Edinburgh, Edinburgh, UK 
Oscar Ribeiro UNIFAI and CINTESIS, ICBAS - University of Porto, Porto, Portugal

Higher Institute of Social Service (ISSSP), Porto, Portugal

University of Aveiro, Aveiro, Portugal

Eric F. Rietzschel University of Groningen, Groningen, The Netherlands

Catherine A. Riffin Department of Human Development, Cornell University, Ithaca, NY, USA

Gail Roberts Australian Catholic University and Villa Maria Catholic Homes, Melbourne, VIC, Australia

Jean-Marie Robine INSERM \& EPHE, Paris and Montpellier, France

Gail A. Robinson Neuropsychology Research Unit, School of Psychology, The University of Queensland, Brisbane, QLD, Australia

Neuropsychology, Department of Neurology, Royal Brisbane and Women's Hospital, Brisbane, QLD, Australia

Natália Pessoa Rocha Laboratório Interdisciplinar de Investigação Médica, Faculdade de Medicina, Universidade Federal de Minas Gerais, Belo Horizonte, MG, Brazil

Maree Roche School of Psychology, University of Waikato, Hamilton, New Zealand

Rachel Rodriguez VA Palo Alto Health Care System, Palo Alto, CA, USA

Jan Philipp Röer Heinrich Heine University Düsseldorf, Düsseldorf, Germany

Nina T. Rogers Department of Epidemiology and Public Health, University College London, London, UK

Alexia Rohde The University of Queensland, St Lucia, Brisbane, QLD, Australia

Anna Rolleston Te Kupenga Hauora Māori, Faculty of Medical and Health Sciences, The University of Auckland, Auckland, New Zealand

David Rooney Macquarie University, Sydney, NSW, Australia

Tanya Rose The University of Queensland, St Lucia, Brisbane, QLD, Australia

Luis Rosero-Bixby University of California, Berkeley, CA, USA

University of Costa Rica, San José, San José, Costa Rica

Kathrin Rosing Psychology of Entrepreneurial Behavior, Institute of Psychology, University of Kassel, Kassel, Germany

Christoph Rott Institute of Gerontology, Heidelberg University, Heidelberg, Germany 
Olivier Rouaud LEAD CNRS UMR 5022, Université de Bourgogne, Dijon, France

University of Burgundy, Dijon, France

CMRR Dijon, Dijon, France

Cort W. Rudolph Saint Louis University, Saint Louis, MO, USA

Clair Rummel VA Puget Sound Health Care System - Seattle Division, Seattle, WA, USA

Eric Ruthruff Department of Psychology, University of New Mexico, Albuquerque, NM, USA

Paul Sacco University of Maryland School of Social Work, Baltimore, MD, USA

Perminder Sachdev Centre for Healthy Brain Ageing, University of New South Wales, Sydney, NSW, Australia

Marian Saeed Department of Surgery, Centre for Eye Research Australia, Royal Victorian Eye and Ear Hospital, Ophthalmology, University of Melbourne, Melbourne, VIC, Australia

Rinat Saifoulline Faculty of Business Administration, University of Applied Sciences, Dresden, Germany

Erin Sakai VA Palo Alto Health Care System, Palo Alto, CA, USA

Karen L. Salekin The University of Alabama, Tuscaloosa, AL, USA

Manisha Salinas Department of Health Promotion and Behavior, College of Public Health, The University of Georgia, Athens, GA, USA

Viktoriya Samarina Barrow Neurological Institute, Phoenix, AZ, USA

Kimberly Sangster Loyola University School of Social Work, Chicago, IL, USA

Kristen Sarkinen School of Nursing, University of Minnesota, Minneapolis, MN, USA

Christine Sattler Department of Industrial and Organizational Psychology, Institute of Psychology, Heidelberg University, Heidelberg, Germany

K. Warner Schaie Department of Psychiatry and Behavioral Sciences, University of Washington, Seattle, WA, USA

Susanne Scheibe Department of Psychology, University of Groningen, Groningen, The Netherlands

Oliver K. Schilling Department of Psychological Ageing Research, Institute of Psychology, Ruprecht-Karls-Universität, Heidelberg, Germany

Hannah Schmitt Saarland University, Saarbrücken, Germany 
Antje Schmitt Department of Business Psychology, Economics and Management and Institute of Psychology, University of Kassel, Kassel, Germany

Eric Schmitt Institute of Gerontology, University of Heidelberg, Heidelberg, Germany

Katharina M. Schnitzspahn School of Psychology, University of Aberdeen, Aberdeen, UK

Jos Schols Maastricht University, Maastricht, The Netherlands

Urte Scholz Department of Psychology, University of Zurich, Zurich, Switzerland

Peter Schönknecht Department of Psychiatry and Psychotherapy, University of Leipzig, Leipzig, Germany

Johannes Schröder Section of Geriatric Psychiatry, Heidelberg University, Heidelberg, Germany

Institute of Gerontology, Heidelberg University, Heidelberg, Germany

Anika Schulz Department of Psychology, University of Groningen, Groningen, Netherlands

Henk Schut Department of Clinical and Health Psychology, Utrecht University, Utrecht, The Netherlands

Benjamin Schüz Division of Psychology, University of Tasmania, Hobart, TAS, Australia

Nadine A. Schwab Department of Clinical and Health Psychology, University of Florida, Gainesville, FL, USA

Forrest Scogin University of Alabama, Tuscaloosa, AL, USA

Theresa L. Scott School of Psychology, The University of Queensland, St Lucia, QLD, Australia

Michael K. Scullin Department of Psychology and Neuroscience, Baylor University, Waco, TX, USA

Jori Sechrist Department of Sociology, McMurry University, Abilene, TX, USA

Geir Selbaek National Norwegian Advisory Unit on Ageing and Health, Vestfold Hospital Trust and Oslo University Hospital, Oslo, Norway

Juan Pedro Serrano Selva Department of Psychology, Faculty of Medicine, University of Castilla-La Mancha, Albacete, Spain

Susan Sharp Memphis Veterans Affairs Medial Center, Memphis, TN, USA

Michael Sharratt Schlegel-University of Waterloo Research Institute for Aging, Kitchener, ON, Canada

Veronica L. Shead South Texas Veterans Health Care System, San Antonio, TX, USA 
Christine Sheppard University of Waterloo, Waterloo, ON, Canada

Bruyère Research Institute, Ottawa, ON, Canada

Yee Lee Shing Center for Lifespan Psychology, Max Planck Institute for Human Development, Berlin, Germany

Jelena S. Siebert Department of Psychological Aging Research, Institute of Psychology, Heidelberg University, Heidelberg, Germany

Mersina Simanski Stanford University, Stanford, CA, USA

David Situ Department of Psychology, California State University, Los Angeles, CA, USA

John Skoumas 1st Cardiology Clinic University of Athens, Athens, Greece

Research Institute for Longevity and Prevention of Geriatric Diseases, Athens, Greece

Glenn E. Smith Mayo Clinic, Rochester, MN, USA

University of Florida College of Public Health and Health Professions, Gainesville, FL, USA

Matthew Lee Smith Department of Health Promotion and Behavior, College of Public Health, The University of Georgia, Athens, GA, USA

Department of Health Promotion and Community Health Sciences, Texas A\&M Health Science Center and School of Public Health, College Station, TX, USA

Jacqui Smith Health and Retirement Study, Survey Research Center, Institute for Social Research, University of Michigan, Ann Arbor, MI, USA

James Smith Rand Corporation, Santa Monica, CA, USA

Amanda Sonnega Health and Retirement Study, Survey Research Center, Institute for Social Research, University of Michigan, Ann Arbor, MI, USA

Rachita Sood University of Miami Miller School of Medicine, Miami, FL, USA

Cyndy G. Soto University of Nevada, Reno, NV, USA

Céline Souchay Laboratoire de Psychologie \& Neurocognition (LPNC), CNRS-UMR 5105, University Grenoble Alpes, Grenoble, France

Laura K. Soulsby School of Psychology, Eleanor Rathbone Building, Liverpool, UK

Anne K. Soutter Department of Management Marketing and Entrepreneurship College of Business and Law, University of Canterbury, Christchurch, New Zealand

Dario Spini Faculty of Social and Political Sciences and Swiss National Centre of Competence in Research LIVES, University of Lausanne, Lausanne, Switzerland 
Ekaterina Staikova Emory University Brain Health Center, Atlanta, GA, USA

Christian Stamov-Roßnagel Jacobs University, Bremen, Germany

John M. Starr Alzheimer Scotland Dementia Research Centre, University of Edinburgh, Edinburgh, Scotland, UK

Ursula M. Staudinger Columbia Aging Center, Columbia University, New York, NY, USA

Allison A. Steen University of Illinois, Urbana, IL, USA

Christodoulos Stefanadis 1st Cardiology Clinic University of Athens, Athens, Greece

Research Institute for Longevity and Prevention of Geriatric Diseases, Athens, Greece

Michelle Steffens School of Psychology, The University of Queensland, Brisbane, QLD, Australia

Andrew Steptoe Department of Epidemiology and Public Health, University College London, London, UK

Harvey L. Sterns The University of Akron, Akron, OH, USA

Brendan Stevenson School of Public Health, Massey University, Palmerston North, New Zealand

Cassandra Stevenson Physical Medicine and Rehabilitation Service, VA Northern California Healthcare System, Martinez, CA, USA

Elizabeth A. L. Stine-Morrow University of Illinois, Urbana, IL, USA

John Strauss School of Economics, University of Southern California, Los Angeles, CA, USA

David L. Strayer The University of Utah, Salt Lake City, UT, USA

Carla M. Strickland-Hughes University of Florida, Gainesville, FL, USA

Margaret Stroebe Department of Clinical and Health Psychology, Utrecht University, Utrecht, The Netherlands

Jessica V. Strong Geriatric Mental Health, VA Boston Healthcare System, Boston, MA, USA

Bonnie Adele Sturrock Department of Surgery, Centre for Eye Research Australia, Royal Victorian Eye and Ear Hospital, Ophthalmology, University of Melbourne, Melbourne, VIC, Australia

Claire Surr Faculty of Health and Social Sciences, Leeds Beckett University, Leeds, UK

Makoto Suzuki Okinawa Research Center for Longevity Science, Urasoe, Okinawa, Japan

Faculty of Medicine, University of the Ryukyus, Okinawa, Japan 
Cassandra Szoeke Department of Medicine - Royal Melbourne Hospital, Consultant Neurologist Department of Neuroscience, Melbourne Health, The University of Melbourne, Parkville, VIC, Australia

Vanessa Taler University of Ottawa and Bruyère Research Institute, Ottawa, ON, Canada

Kristine M. Talley School of Nursing, University of Minnesota, Minneapolis, MN, USA

Sarah (Uma) K. Tauber Department of Psychology, Texas Christian University, Fort Worth, TX, USA

Benjamin Tauber Department of Psychological Aging Research, Institute of Psychology, Heidelberg University, Heidelberg, Germany

Philip Taylor Federation Business School, Federation University Australia, Churchill, VIC, Australia

Ruth Teh Department of General Practice and Primary Health Care, School of Population Health, The University of Auckland, Auckland, New Zealand

Antônio Lúcio Teixeira Laboratório Interdisciplinar de Investigação Médica, Faculdade de Medicina, Universidade Federal de Minas Gerais, Belo Horizonte, MG, Brazil

Laetitia Teixeira UNIFAI and CINTESIS, ICBAS - University of Porto, Porto, Portugal

Adam Theobald Centre for Healthy Brain Ageing, University of New South Wales, Sydney, NSW, Australia

Shane A. Thomas School of Primary Health Care, Monash University, Melbourne, VIC, Australia

International Primary Health Care Research Institute, Shenzhen, China

Susan P. Thompson University of Nevada, Reno, NV, USA

Steven R. Thorp VA San Diego Healthcare System, San Diego, CA, USA

University of California, San Diego, San Diego, CA, USA

Franka Thurm Department of Psychology Chair of Lifespan Developmental Neuroscience, TU Dresden, Dresden, Germany

Eileen C. Toomey Saint Louis University, Saint Louis, MO, USA

Dayna R. Touron University of North Carolina at Greensboro, Greensboro, NC, USA

Andy Towers School of Public Health, Massey University, Palmerston North, New Zealand

Samuel D. Towne, Jr Department of Health Promotion and Behavior, College of Public Health, The University of Georgia, Athens, GA, USA 
Julian Trollor Department of Developmental Disability Neuropsychiatry, School of Psychiatry, University of New South Wales, Sydney, NSW, Australia

Cooperative Research Centre for Living with Autism (Autism CRC), Long Pocket, Brisbane, QLD, Australia

Donald M. Truxillo Department of Psychology, Portland State College of Liberal Arts and Sciences, Portland State University, Portland, OR, USA

Kerstin Unger Department of Neuroscience, Brown University, Providence, RI, USA

Willy Marcos Valencia Geriatrics Research, Education and Clinical Center (GRECC), Miami VA Medical Center, Miami, FL, USA

Department of Public Health Sciences, University of Miami Miller School of Medicine, Miami, FL, USA

S. P. J. van Alphen Department of Clinical and Life Span Psychology, Vrije Universiteit Brussel (VUB), Brussels, Belgium

Silvia D. M. van Dijk University Center for Psychiatry, University Medical Center Groningen, Groningen, The Netherlands

Beatrice Van der Heijden Institute for Management Research, Radboud University, Nijmegen, The Netherlands

Open University of the Netherlands, Heerlen, The Netherlands

Katie Van Moorleghem Palo Alto University, Palo Alto, CA, USA

Sierra Pacific Mental Illness Research Education and Clinical Center, VA Palo Alto Health Care System, Palo Alto, CA, USA

Nico W. Van Yperen Department of Psychology, University of Groningen, Groningen, The Netherlands

Hilde Verbeek Department of Health Services Research, Faculty of Health, Medicine and Life Science, CAPHRI School for Public Health and Primary Care, Maastricht University, Maastricht, The Netherlands

Paul Verhaeghen Georgia Institute of Technology, Atlanta, GA, USA

Frans R. J. Verhey School for Mental Health and Neuroscience, Alzheimer Center Limburg, Maastricht University Medical Center, Maastricht, The Netherlands

Dominique Verté Vrije Universiteit Brussel/ University College Ghent, Brussels, Belgium

Eimee Villanueva Palo Alto University, Palo Alto, CA, USA

Manuel C. Voelkle Institute of Psychology, Humboldt University Berlin, Berlin, Germany

Max Planck Institute for Human Development, Berlin, Germany 
Deborah Vollmer Dahlke Health Promotion and Community Health Sciences, Texas A\&M Health Science Center and School of Public Health, College Station, TX, USA

Hans-Werner Wahl Department of Psychological Aging Research, Institute of Psychology, Heidelberg University, Heidelberg, Germany

Network Aging Research (NAR), Heidelberg University, Heidelberg, Germany

Tomoko Wakui Tokyo Metropolitan Institute of Gerontology, Tokyo, Japan

Katherine E. Walesby Alzheimer Scotland Dementia Research Centre and Centre for Cognitive Ageing and Cognitive Epidemiology, University of Edinburgh, Edinburgh, Scotland, UK

Nicole Walker School of Psychology, The University of Queensland, Brisbane, QLD, Australia

Ruth V. Walker The University of Akron, Akron, OH, USA

Sarah J. Wallace The University of Queensland, St Lucia, Brisbane, QLD, Australia

Wylie H. Wan Oregon Health and Science University, Portland, OR, USA

Anne Wand School of Psychiatry, University of NSW, Sydney, NSW, Australia

South East Sydney Local Health District, Sydney, NSW, Australia

Yafeng Wang Institute of Social Science Survey, Peking University, Beijing, China

Daniela Weber World Population Program, Wittgenstein Centre for Demography and Global Human Capital, International Institute for Applied Systems Analysis, Laxenburg, Austria

Jennifer C. Weeks Department of Psychology, University of Toronto, Toronto, ON, USA

Rotman Research Institute, Baycrest, Toronto, ON, USA

Matthias Weigl Institute and Outpatient Clinic for Occupational, Social, and Environmental Medicine, Ludwig-Maximilians-University, Munich, Germany

Stephanie Y. Wells VA San Diego Healthcare System, San Diego, CA, USA University of California, San Diego, San Diego, CA, USA

Yvonne Wells La Trobe University, Melbourne, VIC, Australia

Kathleen A. Welsh-Bohmer Departments of Psychiatry and Neurology, Duke University Medical Center, Durham, NC, USA

Britta Wendelstein Section of Geriatric Psychiatry, Heidelberg University, Heidelberg, Germany

Institute of Gerontology, Heidelberg University, Heidelberg, Germany 
Robert West Department of Psychology, Iowa State University, Ames, IA, USA

Robin L. West University of Florida, Gainesville, FL, USA

Markus Wettstein Department of Psychological Aging Research, Institute of Psychology, Heidelberg University, Heidelberg, Germany

Network Aging Research (NAR), Heidelberg University, Heidelberg, Germany

Bradley Willcox Okinawa Research Center for Longevity Science, Urasoe, Okinawa, Japan

Hawaii Lifespan and Healthspan Studies, Kuakini Medical Center, Honolulu, HI, USA

Department of Geriatric Medicine, John A. Burns School of Medicine, University of Hawaii, Honolulu, HI, USA

The Queen's Medical Center, Honolulu, HI, USA

D. Craig Willcox Okinawa Research Center for Longevity Science, Urasoe, Okinawa, Japan

Department of Human Welfare, Okinawa International University, Ginowan, Okinawa, Japan

Hawaii Lifespan and Healthspan Studies, Kuakini Medical Center, Honolulu, HI, USA

Department of Geriatric Medicine, John A. Burns School of Medicine, University of Hawaii, Honolulu, HI, USA

Sherry L. Willis Department of Psychiatry and Behavioral Sciences, University of Washington, Seattle, WA, USA

Tim D. Windsor Flinders University, Adelaide, SA, Australia

Barbara Wisse Department of Psychology, University of Groningen, Groningen, Netherlands

Amber E. Witherby Department of Psychology, Texas Christian University, Fort Worth, TX, USA

Oliver T. Wolf Department of Cognitive Psychology, Institute of Cognitive Neuroscience, Ruhr University Bochum, Bochum, Germany

Gloria Wong The University of Hong Kong, Hong Kong, China

Linda Worrall The University of Queensland, St Lucia, Brisbane, QLD, Australia

Camille B. Wortman Department of Psychology, SUNY Stony Brook, Stony Brook, NY, USA 
Sarah Wright Department of Management Marketing and Entrepreneurship College of Business and Law, University of Canterbury, Christchurch, New Zealand

Carsten Wrosch Concordia University, Montreal, QC, Canada

Susanne Wurm Institute of Psychogerontology, Friedrich-Alexander Universität Erlangen, Nürnberg (FAU), Germany

Jean F. Wyman School of Nursing, University of Minnesota, Minneapolis, MN, USA

Lale M. Yaldiz Department of Psychology, Portland State University, Portland, OR, USA

Zixuan Yang Centre for Healthy Brain Ageing, University of New South Wales, Sydney, NSW, Australia

Melissa A. Yanovitch PGSP-Stanford PsyD Consortium, Palo Alto, CA, USA

Mônica Sanches Yassuda University of São Paulo, São Paulo, SP, Brazil

Dannii Y. Yeung Department of Applied Social Sciences, City University of Hong Kong, Hong Kong, China

Brian Yochim Department of Medicine, National Jewish Health, University of Colorado School of Medicine, Denver, CO, USA

Carmen K. Young Department of Psychology, Rice University, Houston, TX, USA

Hannes Zacher Department of Psychology, University of Groningen, Groningen, The Netherlands

Sara Zaniboni Department of Psychology and Cognitive Science, Università degli studi di Trento, Rovereto, TN, Italy

Yi Zeng Center for the Study of Aging and Human Development and Geriatrics Division, School of Medicine, Duke University, Durham, NC, USA

Center for Healthy Aging and Development Studies, National School of Development, Peking University, Beijing, China

Andreas Zenthöfer Department of Prosthodontics, University Hospital Heidelberg, Heidelberg, Germany

Yaohui Zhao National School of Development, Peking University, Beijing, China

Hanna Zieschang Institute for Work and Health of the German Social Accident Insurance, Dresden, Germany

Daniel Zimprich Department of Psychology, Ulm University, Ulm, BadenWürttemberg, Germany 
